\title{
Synthesis of 2-tetrafluoropyridyl-4,5-disubstituted 1,2,3-triazoles
}

\author{
Khalil Beyki*, Malek Taher Maghsoodlou and Reza Heydari
}

\begin{abstract}
By cycloaddition reaction of sodium azide with chalcone in the presence of $\mathrm{CuO}$ as a catalyst in DMF a 1,2,3-triazole are prepared in reaction with pentafluoropyridine to give 2-(tetrafluoropyridin-4-yl)-1,2,3-triazole derivatives in good yields and high regioselectivity. The regioselectivity of the compounds are confirmed by ${ }^{19} \mathrm{~F}-\mathrm{NMR}$ and other spectroscopy.
\end{abstract}

Keywords: Pentafluoropyridine, Chalcone, 1,2,3-Triazoles, CuO, ${ }^{19} \mathrm{~F}-\mathrm{NMR}$

\section{Background}

Recently, perfluorocarbons have been used as building blocks in the pharmaceutical industry and in material science due to their unique properties (Kenneth 2006; Bruce 2001). In pharmacology and medicinal researches, it is common to substitute hydrogen with fluorine atom for increasing the physiochemical (e.g. solubility, stability) and biological activity (e.g. absorption, distribution, metabolism, elimination and toxicity) of drugs (Iwao 2009).

Pentafluoropyridine in which all the hydrogen atoms in pyridine ring have been replaced by fluorine atoms are highly susceptible towards nucleophilic attack owing to the presence of several highly electronegative fluorine atoms and nitrogen hetero atom; consequently, the chemistry of pentafluoropyridine is dominated by nucleophilic aromatic substitution processes and new chemistry continues to emerge (Iwao 2009; Reza et al. 2008; Mark et al. 2013; Van Ba and Donald 2012). The order of nucleophilic attack for pentafluoropyridine is established to be para > ortho > meta positions, so the reactions of pentafluoropyridine with some nucleophile occur selectively at the para position as this site is most activated towards nucleophilic additions to afford of 4-substited

\footnotetext{
*Correspondence: chemkhalil@pgs.usb.ac.ir

Department of Chemistry, Faculty of Science, University of Sistan and Baluchestan, P.O. Box 98135-674, Zahedan, Iran
}

tetrafluoropyridine (Hadjar et al. 2001; Matthew et al. 2010; Jingjing et al. 2014).

Baohua Chen and coworker's reported the synthesis of $\mathrm{N}$-2-aryl-substituted-1,2,3-triazoles and arylation in the last step (Yuanqing et al. 2012). With this goal, we have employed the highly electron-deficient pentafluoropyridine for arylation of 1,2,3-triazoles in the last step for preparation of 2-tetrafluoropyridyl-1,2,3 triazoles (Fig. 1).

Earlier, we reported the synthesis of 4-substituted2,3,5,6-tetrafluoropyridine derivatives by the reaction of pentafluoropyridine with malononitrile, 1-tetrazole5-thiol, piperazine (Khalil et al. 2015), hydroxylated naphtoquinones and hydroxylated antraquinones (Khalil et al. 2016).

\section{Results and discussion}

In this research, we describe the synthesis of 2-tetrafluoropyridyl-4,5-disubstituted-1,2,3-triazoles via Diels-Alder cycloaddition reaction of sodium azide and 1,3-diphenylprop-2-en-1-one (chalcone) and then nucleophilic substitution reactions with pentafluoropyridine in DMF as solvent.

Reaction of 3-(phenyl)-1-phenylprop-2-en-1-one (chalcone) (1a) with sodium azide and one equiv catalyst of $\mathrm{CuO}$ in the solvent of DMF gave intermediate of 4-benzoyl-5-phenyl-1,2,3-triazol-2-ide 3 (TLC monitoring during the course of the reaction; Fig. 2). In last step, nitrogen nucleophile of 1,2,3-triazoles (intermediate 3) attack at the most activated 4-position of 


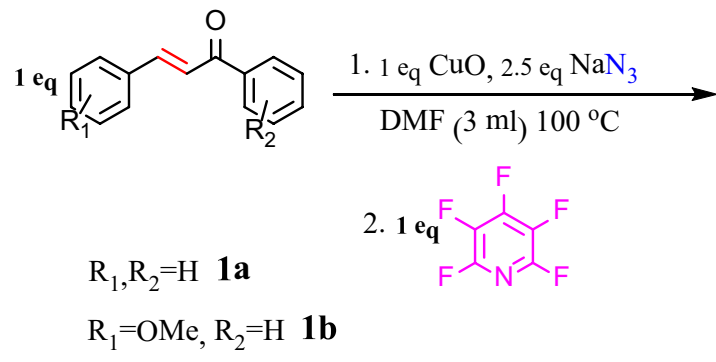<smiles></smiles>

Fig. 1 Synthesis of 2-tetrafluoropyridyl-1,2,3-triazoles

pentafluoropyridine and elimination of 4-fluor pyridine ring leads to the formation of (2-(perfluoropyridin-4-yl)5-phenyl-2H-1,2,3-triazol-4-yl)(phenyl)methanone 2a in good yield. In the mechanism of this transformation $\mathrm{CuO}$ acted as the oxidant and then $\mathrm{Cu}$ was oxidized to $\mathrm{CuO}$ by air. In this search, we did not perform any optimization for this protocol and utilize the same condition reported in previous paper (Yuanqing et al. 2012).

Purification of 2a was achieved by column chromatography using ethyl acetate/ $n$-hexane $(2: 10)$. The melting point, ${ }^{19} \mathrm{~F},{ }^{1} \mathrm{H},{ }^{13} \mathrm{C}$ NMR and mass spectra of the obtained product clearly indicated the formation of (2-(perfluoropyridin-4-yl)-5-phenyl-2H-1,2,3-triazol-4-yl)(phenyl) methanone 2a. For example, in the ${ }^{1} \mathrm{H}$ NMR spectrum of compound $2 \mathrm{a}$, the aromatic proton resonances were observed as multiplets at $\delta=7.60-8.61 \mathrm{ppm}$. The ${ }^{13} \mathrm{C}$ NMR spectrum of compound 2a showed 20 distinct resonances consistent with the recommended structure.

In ${ }^{19} \mathrm{~F}$ NMR analyze of $2 \mathbf{a}$ exhibited two peaks for fluorine's, a peak is observed at down field of doublet of doublet at $\delta=-89.54(\mathrm{~J}=24,28 \mathrm{~Hz})$ for $\mathrm{F}-2,6$ (ortho positions) and also, a doublet of doublet is remarked at up field $-144.83(J=20,8 \mathrm{~Hz})$ for $\mathrm{F}-3,5$ (meta positions). A part of the ${ }^{19} \mathrm{~F}$ NMR spectrum of $\mathbf{2 a}$ is shown in Fig. 3. ${ }^{19} \mathrm{~F}$ NMR analysis of $2 \mathrm{a}$ confirmed that the nucleophilic substitution had occurred at the 4-position of pyridine ring. The mass spectrum of 2a displayed molecular ion peak $\left(\mathrm{M}^{+}\right)$at $m / z=399$, which is consistent with the proposed structure. Other ion peak are shown in mass spectra of 2a (Fig. 4).

The structure of compounds $2 \mathbf{b}$ was confirmed by NMR spectroscopic data. In particular, ${ }^{19} \mathrm{~F}-\mathrm{NMR}$ spectroscopy show the chemical shift of fluorine atoms attached to the ortho and meta position are observed respectively at -95 and $-153 \mathrm{ppm}$. The ${ }^{1} \mathrm{H}$ NMR spectra of compound $\mathbf{2 b}$ showed an $\mathrm{H}$ broad signal at $4.5 \mathrm{ppm}$ for $\mathrm{OCH}_{3}$ group, and the protons of the phenyl ring were observed at $\delta=7.6-8.6 \mathrm{ppm}$. The mass spectrum of $\mathbf{2 b}$ displayed the molecular ion peak $(M-1)$ at $m / z=427$, which is consistent with the proposed structure.

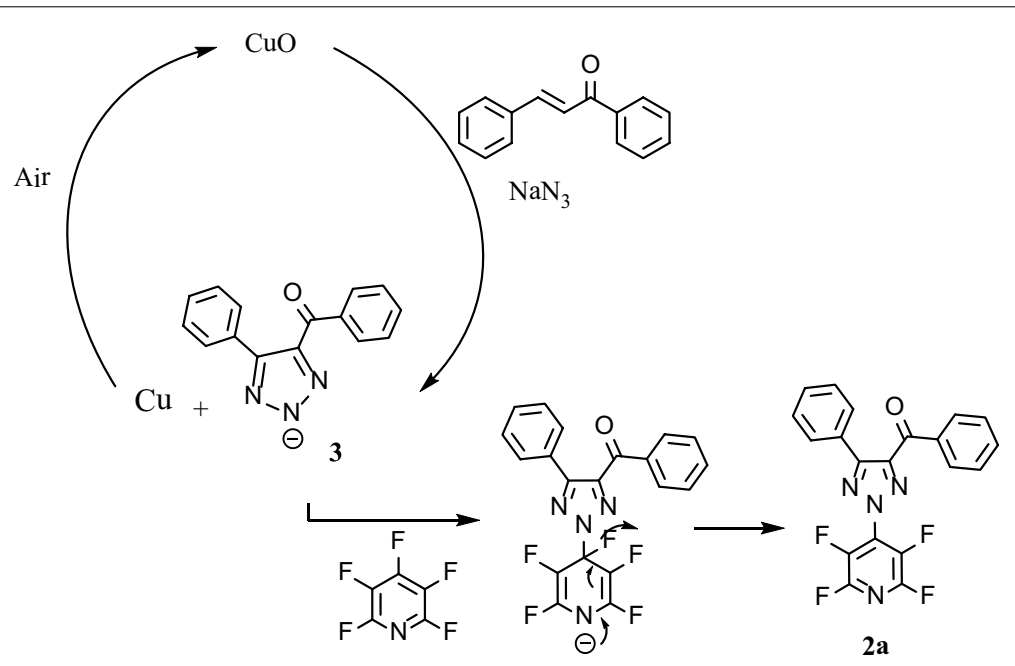

Fig. 2 The mechanism for the formation of 2-tetrafluoropyridyl-4,5-disubstituted 1,2,3-triazoles 


\section{Experimental}

All reagents and solvents were purchased from Aldrich and Merck were used without further purification. The ${ }^{1} \mathrm{H}$ and ${ }^{13} \mathrm{C}$ NMR spectra were obtained on Bruker with DMSO as a solvent $\left({ }^{1} \mathrm{H}\right.$ NMR at $300 \mathrm{MHz}$ and ${ }^{13} \mathrm{C}$ NMR at $75 \mathrm{MHz})$. In the ${ }^{19} \mathrm{~F}-\mathrm{NMR}$ spectra $(282 \mathrm{MHz})$, up field shifts were quoted as negative and referenced to $\mathrm{CFCl}_{3}$. Mass spectra were taken by a Micro mass Platform II: EI mode $(70 \mathrm{eV})$.

\section{General procedure for the preparation}

\section{of 2-(tetrafluoropyridin-4-yl)-1,2,3-triazole}

A mixture of chalcone $(1 \mathrm{mmol})$, sodium azide $(1 \mathrm{mmol})$ and $\mathrm{CuO}(2.5 \mathrm{~mol} \%)$ were stirred in DMF $(3 \mathrm{~mL})$ for $20 \mathrm{~h}$ at $100{ }^{\circ} \mathrm{C}$. After completion of the reaction as indicated

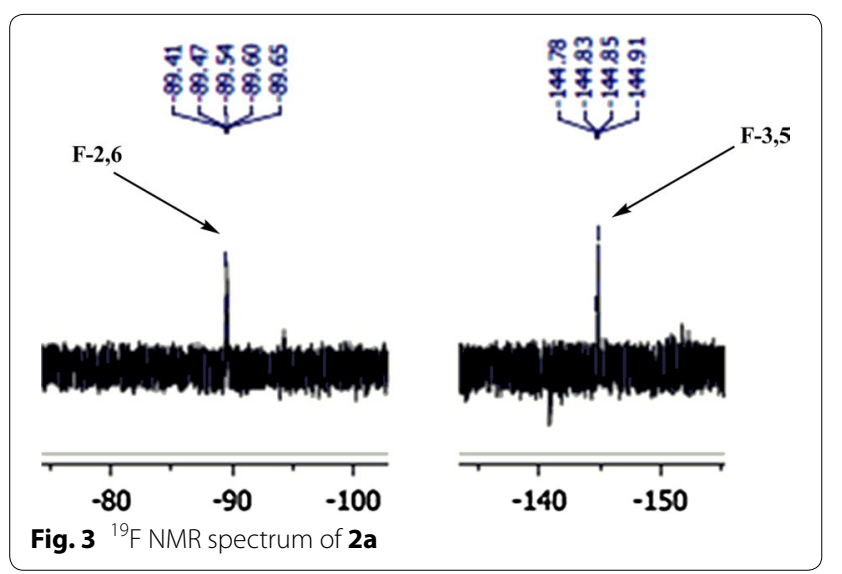

by TLC pentafluoropyridine ( $1 \mathrm{mmol}$ ) was added to the mixture and the reaction continued at $100{ }^{\circ} \mathrm{C}$ for $5 \mathrm{~h}$. Following, to the reaction mixture was added water $4 \mathrm{~mL}$, and extracted with ethyl acetate and dichloromethane $(3 \times 5 \mathrm{~mL})$. The solvent was removed in vacuo, and the crude product was purified by column chromatography using ethyl acetate/ $n$-hexane $(2 / 10)$ to give the pure product.

\section{(2-(Perfluoropyridin-4-yl)-5-phenyl-2H-1,2,3-triazol-}

4-yl)(phenyl)methanone (2a)

(0.24 g, 65 \%) as brown solid; $\mathrm{mp} 240-245{ }^{\circ} \mathrm{C}$ decompose.

${ }^{1} \mathrm{H}$ NMR (DMSO): $\delta$ (ppm) 7.96-8.41 (10H, m, Ar-H);

${ }^{19} \mathrm{~F}$ NMR (DMSO): $\delta$ (ppm) -89.5 (2F, m, J J $\left.23, \mathrm{~F}-2,6\right)$, -144.8 (2F, m, J $\left.\mathrm{FF}_{\mathrm{FF}} 20, \mathrm{~F}-3,5\right) ;{ }^{13} \mathrm{C}$ NMR (DMSO): $\delta$ (ppm) $126.81,126.96,127.40,128.14,128.18,128.24,128.29$, $128.66,128.74,128.86,128.92,129.23,129.44,129.52$, $129.77,129.86,170.31 \mathrm{ppm}$. MS (EI), $m / z(\%)=415$ $\left[\mathrm{M}+\mathrm{NH}_{3}\right]^{+}, 399(\mathrm{M}), 384,360,343,316,286,268,248$, 220, 199, 169, 141, 119, 101, 86, 58, 42.

\section{(5-(4-Methoxyphenyl)-2-(perfluoropyridin-4-yl)-} 2H-1,2,3-triazol-4-yl)(phenyl)methanone (2b)

$(0.20 \mathrm{~g}, 60 \%)$ as a yellow solid; mp $265-270{ }^{\circ} \mathrm{C}$ decompose. ${ }^{1} \mathrm{H}$ NMR (DMSO): $\delta(\mathrm{ppm}) 4.5\left(3 \mathrm{H}, \mathrm{OCH}_{3}\right) 7.60$ 8.61 (10H, m, Ar-H); ${ }^{19} \mathrm{~F}$ NMR (DMSO): $\delta$ (ppm) -95.0 (2F, m, F-2,6), -153.0 (2F, m, F-3,5); ${ }^{13} \mathrm{C}$ NMR (DMSO): $\delta(\mathrm{ppm}) 114.29,115.54,121.54,122.94,123.23,127.82$, $130.35,130.85,140.85,141.22,157.44 \mathrm{ppm}$. MS (EI), $\mathrm{m} / \mathrm{z}$ $(\%)=427[M-1], 321,282,165,91$.

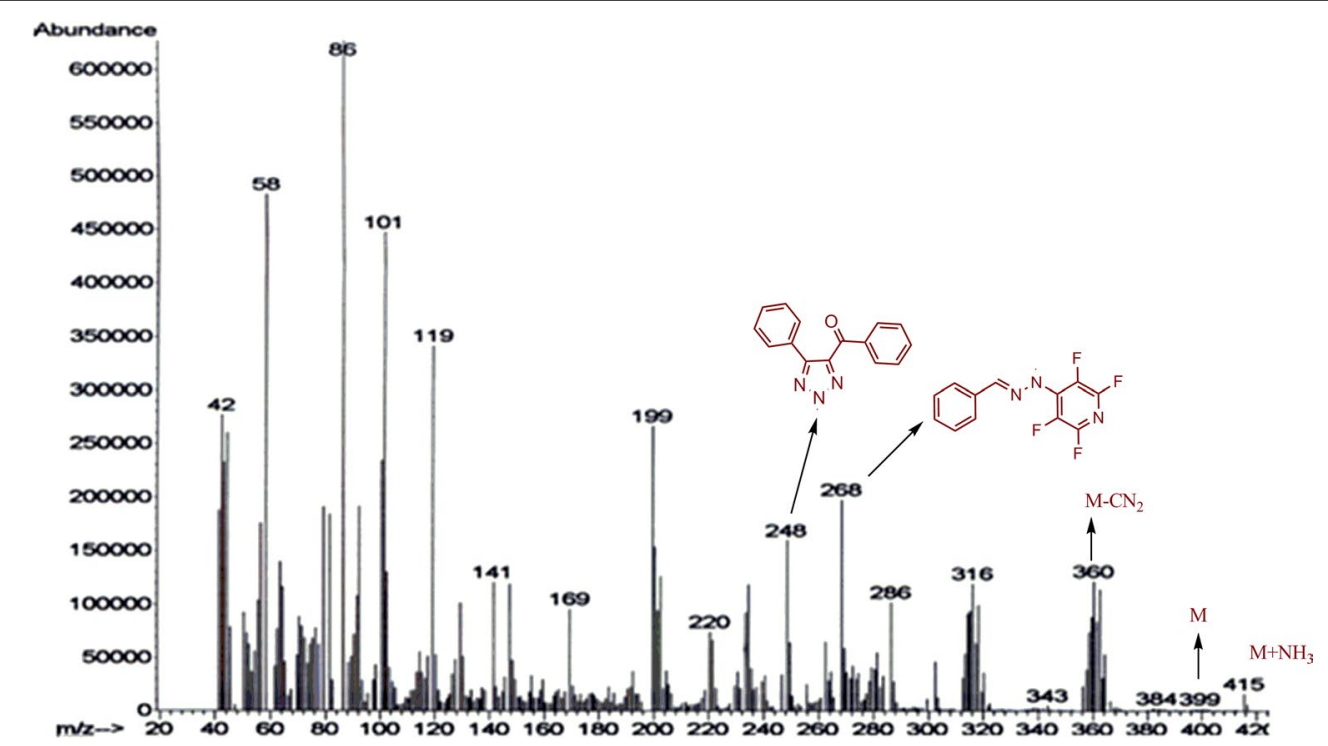

Fig. 4 Mass spectra of $\mathbf{2 a}$ 


\section{Conclusion}

Diels-Alder cycloaddition reaction of chalcone with azide in the presence of $\mathrm{CuO}$ as catalyst gives 4,5-disubstituted 1,2,3-triazoles, in reaction with pentafluoropyridine give 4,5-disubstituted-2 tetrafluoropyridyl-1,2,3-triazoles.

\section{Authors' contributions}

Acquisition of data: from articles and books: Analysis and interpretation of data: by RH and KB. Drafting of manuscript: KB. Critical revision: MTM. All authors read and approved the final manuscript.

\section{Acknowledgements}

The authors gratefully acknowledge partial support of this work by the Research Affairs Office of Sistan and Balouchestan University.

\section{Competing interests}

The authors declare that they have no competing interests.

Received: 3 June 2016 Accepted: 27 September 2016

Published online: 11 November 2016

\section{References}

Van Ba N, Donald JB (2012) Preparation of p-substituted tetrafluoropyridyl derivatives via the tetrafluoropyridylcopper reagent. J Fluor Chem 135:144-154

Bruce ES (2001) Fluorine substituent effects (on bioactivity). J Fluor Chem 109:3-11
Hadjar B, Richard DC, Philip RH, Graham S (2001) Multi-substituted heterocycles. J Fluor Chem 112:133-137

Iwao O (2009) Fluorine in medicinal chemistry and chemical biology. Blackwell, Oxford

Jingjing Wu, Dan F, Song C (2014) Synthesis of polyfluoroaryl-containing 1,2,3-triazoles by reaction of polyfluoroarenes, sodium azide and active methylene ketones/esters. J Fluor Chem 168(2014):230-235

Kenneth LK (2006) Fluorine in medicinal chemistry: recent therapeutic applications of fluorinated small molecules. J Fluor Chem 127:1013-1029

Khalil B, Reza H, Malek Taher M (2015) Synthesis of 2,3,5,6-tetrafluoro-pyridine derivatives from reaction of pentafluoropyridine with malononitrile, piperazine and tetrazole-5-thiol. SpringerPlus 4:757

Khalil B, Reza H, Malek Taher M (2016) Reaction of hydroxylated naphtoquinones/antraquinones with pentafluoropyridine. SpringerPlus 5:110

Mark AF, Graham P, Graham S, Andrei SB (2013) ${ }^{19} \mathrm{~F}$ and ${ }^{13} \mathrm{C}$ GIAO-NMR chemical shifts for the identification of perfluoro-quinoline and-isoquinoline derivatives. J Fluor Chem 155:62-71

Matthew WC, Emma LP, Graham P, Rachel S, Graham S, Ian W, Dmitrii SY, Judith AKH, John AC, David DM (2010) Annelation of perfluorinated heteroaromatic systems by 1,3-dicarbonyl derivatives. Tetrahedron 66(2010):3222-3227

Reza RK, Graham S, Dmitrii SY, Judith AKH (2008) Macrocycles from pentafluoropyridine and tetrafluoropyrimidine. J Fluor Chem 129:307-313

Yuanqing Z, Xiaolong L, Jihui L, Jinying C, Xu M, Mingming Z, Baohua C (2012) $\mathrm{CuO}$-promoted construction of $\mathrm{N}$-2-aryl-substituted-1,2,3-triazoles via azide-chalcone oxidative cycloaddition and post-triazole arylation. Org Lett 14(1):26-29

\section{Submit your manuscript to a SpringerOpen ${ }^{\odot}$ journal and benefit from:}

- Convenient online submission

- Rigorous peer review

- Immediate publication on acceptance

- Open access: articles freely available online

- High visibility within the field

- Retaining the copyright to your article 